\title{
Effect of Carrying Weight on the Gait of Elderly Women when using a Walking Assistant Vehicle
}

\author{
Hyo-Lyun Roh, PT, PhD • Sung-Min Son, OT, MS ${ }^{1}$ Sung-Won Kwag, OT, $\mathrm{MS}^{1 \dagger}$
}

Department of Physical Therapy, Kangwon National University

${ }^{1}$ Department of Emergency Medical Service Rehabilitation, Kangwon National University Graduate School

Received: June 15, 2018 / Revised: June 18, 2018 / Accepted: August 6, 2018

(c) 2018 J Korean Soc Phys Med

\section{| Abstract |}

PURPOSE: This study was conducted to analyze the effects of carrying weight on the gait of elderly women using a walking-assistant vehicle (WAV) as the weight increased.

METHODS: A total of 30 elderly women living in the local community were included as subjects and instructed to walk $50 \mathrm{~m}$ using a WAV loaded with sandbags corresponding to $0 \%, 5 \%, 10 \%$, or $15 \%$ of their mean weight. The subjects' gait was analyzed using a gait analyzer to measure stride length, step length, step width, and gait time.

RESULTS: Stride and step lengths were longest when carrying $5 \%$ of their weight and shortest when carrying $15 \%$ of their mean body weight. Step width and gait time were lowest when carrying weights corresponding to $5 \%$ and highest for $15 \%$ of their mean body weight. When observing gait with a WAV, the gait time was greatly affected by weights, with carrying weight equivalent to $5 \%$ of the body

$\dagger$ Corresponding Author : Sung-Won Kwag eroom5557@naver.com, http://orcid.org/0000-0003-4700-8387 This is an Open Access article distributed under the terms of the Creative Commons Attribution Non-Commercial License (http://creativecommons.org/licenses/by-nc/3.0) which permits unrestricted non-commercial use, distribution, and reproduction in any medium, provided the original work is properly cited. weight positively affected the gait with a WAV, whereas carrying weights of $>15 \%$ resulted in slower gait speed.

CONCLUSION: When walking with a WAV, an appropriate carrying weight of approximately $5 \%$ of the body weight stabilizes gait, while a weight of $15 \%$ leads decreased gait efficiency. Therefore, when using a WAV during outdoor activities, elderly women should add some weight to the WAV; however, the carrying weight should be $<15 \%$ of the body weight.

Key Words: Elderly women, Gait, Walking assistant

\section{Introduction}

In humans, physical ability declines with age, and this decline is particularly severe in gait because of reduced bone density resulting from a decrease in bone mass due to aging-related loss of intraosseous calcium. This increases the risk of fracture among elderly individuals (Park, 2018). This risk is especially great in the case of elderly women because of the reduction of postmenopausal secretion of the sex hormone estrogen, which helps to maintain the bone density with the risk of the facture; accordingly, the prevalence of the gait impairment is greater in elderly women than elderly men. Indeed, a report by the Korea Health Industry Development Institute in 2001 indicated 
that the prevalence of the gait impairment according to low back pain and osteoporosis for elderly women was found to be more than twice that of elderly men (Chang, 2001).

Therefore, various walking aids have been developed and used to relieve difficulties with gait in elderly women (Baek, 2012). Here, WAVs improve stability by supporting the upper body during gait, maintaining safe gait by assisting balance and trunk movement, and effectively preventing falls (Jung et al., 2009). Moreover, elderly women live longer than men, have a higher demand for leisure activities (Park, 2017), and their usage of WAVs is continually increasing. Designed in the form of strollers that can be used for shopping or long-distance walking with space to carry items and provide the ability to sit and rest, WAVs have become established as a must-have walking aid for elderly women during outdoor activities (Park and Cho, 2008).

However, inappropriate use of WAVs by elderly women can introduce significant risks. In particular, carrying excessive luggage is an important factor that can have a negative physical effect on the walker, altering his or her gait pattern. The excessive force required when carrying too much weight increases physical burden and center of mass and trunk movements, which in turn elevates the risk of gait impairment due to reduced ability to maintain balance. Thus, through the contribution of the WAV instability, the risk of overturning increases, which presents considerable danger to elderly individuals during walking (Shin et al., 2015). For this reason, the carrying weight should be considered when using WAVs (Park and Cho, 2008).

Various studies of WAVs have been conducted, including studies to improve design through anthropometric analysis and usability assessment (Jung et al., 2009), to improve design for better stability (Cho et al., 2011), and to improve product quality and function to better reflect the needs of the elderly. In addition, studies have investigated the detection of dangerous situations with WAVs (Lee et al., 2013), and to compare gait stability between individuals using a WAV and a stroller as a walking aid (Park et al., 2009). The gait pattern according to position and weight when using a walker (Shin et al., 2015) and with an external load applied (Roh and Shin, 2015) have already been analyzed. However, research on walking aids and weight loading has been limited.

Hence, to increase gait stability and reduce the risk of safety accidents, this study was designed to test the hypothesis that increasing the carrying weight when using a WAV changes the gait of the elderly women. To accomplish this, the effects of increasing weight on the gait of the elderly women when using WAVs were measured to help determine the appropriate carrying weight when using WAVs.

\section{Methods}

The subjects consisted of 30 healthy elderly women aged $\geq 65$ years who used the elderly welfare center in K City in Korea.

Only individuals who 1 . were healthy adults 2 . received a Mini Mental Status Examination-Korean Version (MMSE-K) score of at least 24 points, which corresponds to "normal" in the criteria for cognitive level, 3. had no problem with physical structures and function, and 4 . had not been diagnosed with muscular skeletal or neurological diseases that would cause problems with gait were included. Moreover, only individuals who already had experience in the use of WAVs participated in this study. The exclusion criteria were as follows: The people who had no visual or auditory impairment causing a problem with gait, history of gait impairment, taking any antipsychotic medication, and wearing a gait aid due to physical structure problems.

Gait analysis was conducted by two occupational therapists under the supervision of one professor specializing in physiotherapy. Gait analysis was separately 


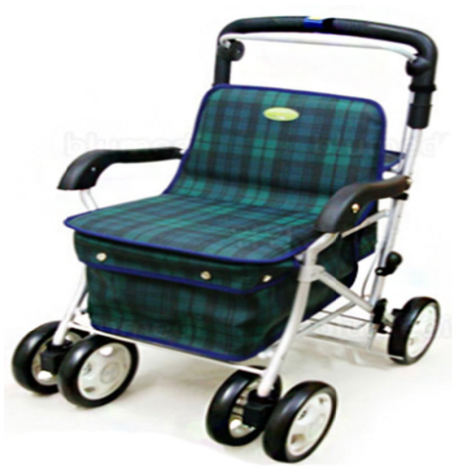

Fig 1. The walking assistant vehicle

conducted for each subject in the elderly welfare center auditorium under calm, tranquil conditions, after cleaning the surrounding environment. All subjects received sufficient explanation about the objectives of the study before the experiment and provided their consent to participate. This study was reviewed and approved by the Kangwon National University Institutional Review Board (KWNUIRB-2016-03-008-001), and all subjects signed an informed consent form.

For the WAV (CareMax inc., Taiwan), we selected the model with the highest preference and rate of purchase among elderly women that had space to carry luggage and could also be used as a chair. This WAV also has an adjustable handle height and brakes (Chang, 2001). The WAV was made of aluminum, PVC, and fabric, with overall dimensions of $490 \times 640 \times 830-910 \mathrm{~mm}$, a chair width of 360 $\mathrm{mm}$, a chair height of $430 \mathrm{~mm}$, and a weight of $6 \mathrm{~kg}$ (Fig. 1). During gait, the WAV handle was adjusted to the most efficient height, at the greater trochanter of the femur, allowing the elbow to be flexed to an angle of 15-20 (Roh and Shin, 2015).

A WalkWay MG-1000 (Animaco, Japan) walking analyzer was used to measure the step length, stride length, step width, and time according to storage weight. First, the physical properties of the subjects were measured. All subjects wore casual clothes and socks without shoes for the measurements. Before the experiment, the subjects underwent sufficient stretching and a 5-minute walking exercise using the WAV to familiarize themselves with walking with the WAV. The experiment began when the subjects could maintain natural walking. For accurate measurement of gait, subjects began walking from $3 \mathrm{~m}$ before the start of the gait analyzer and walked naturally for $50 \mathrm{~m}$ with the WAV positioned in the center of the gait analyzer. During gait analysis, the weight was adjusted by loading sandbags into the luggage-carrying compartment that had weights equivalent to $0 \%, 5 \%, 10 \%$, or $15 \%$ of the subjects' mean body weight. Therefore, each subject walked four times.

The data collected for each variable were encoded and analyzed using SPSS ver. 18. The subjects' general characteristics were analyzed using descriptive statistics and one-way ANOVA was used to identify significant differences according to weight while using the WAV, with Duncan's post hoc tests employed to verify between-group differences. To determine the effects of age and carrying weight on gait, we performed a simple regression. The statistical significance was $95 \%$.

\section{Results}

\section{General characteristics of the subjects}

When analyzing the subjects' general characteristics, 14 $(46.70 \%)$ of the 30 elderly women were aged $65^{-74}$ years, making it the most common age group. The mean height and body weight were $154.00 \pm 5.39 \mathrm{~cm}$ and $62.73 \pm 6.24$ $\mathrm{kg}$, respectively (Table 1 ).

\section{Gait analysis according to storage weight}

Gait was analyzed when using the WAV carrying no weight or weight equal to $5 \%, 10 \%$, or $15 \%$ of the mean weight of the subjects. Stride length showed statistically significant differences according to carrying weight $(\mathrm{p}<.001)$, with the longest stride length of $77.19 \pm 17.47 \mathrm{~cm}$ occurring at a carrying weight of $5 \%$, and the shortest 
Table 1. General Characteristics

\begin{tabular}{|c|c|c|c|c|}
\hline \multirow{2}{*}{ N (\%) } & \multicolumn{2}{|c|}{ Age } & \multirow{2}{*}{ Height (cm) } & \multirow{2}{*}{ Weight $(\mathrm{kg})$} \\
\hline & Years & N (\%) & & \\
\hline \multirow{3}{*}{$30(100 \%)$} & $65-74$ & $14(46.70 \%)$ & & \\
\hline & $75-84$ & $10(33.70 \%)$ & $154.00 \pm 5.39$ & $62.73 \pm 6.24$ \\
\hline & 85 Above & $6(20.00 \%)$ & & \\
\hline
\end{tabular}

stride length of $55.79 \pm 22.11 \mathrm{~cm}$ occurring at a carrying weight of $15 \%$. Post hoc testing confirmed that the $15 \%$ group was significantly different from those of the $0 \%$, $5 \%$, and $10 \%$ groups. Step length also showed significant differences according to carrying weight $(\mathrm{p}<.001)$, with the longest step length of $37.90 \pm 10.59 \mathrm{~cm}$ occurring at a carrying weight of $5 \%$ and the shortest step length of $26.91 \pm 7.67 \mathrm{~cm}$ occurring at a carrying weight of $15 \%$. Post hoc tests verified that the $15 \%$ group was significantly different from those of the $0 \%, 5 \%$, and $10 \%$ groups.

Step width showed significant differences according to carrying weight $(p<.001)$, with the shortest step width of $11.48 \pm 2.14 \mathrm{~cm}$ being observed for a carrying weight of $5 \%$ and the longest step width of $16.75 \pm 2.15 \mathrm{~cm}$ being observed for a carrying weight of $15 \%$. Post hoc testing verified that the step width of the $15 \%$ group was significantly different from that of the $0 \%, 5 \%$, and $10 \%$ groups. Gait time showed statistically significant differences according to carrying weight $(p<.001)$, with the fastest gait time of $2.23 \pm .45 \mathrm{sec}$ being observed for a carrying weight of $5 \%$ and the slowest gait time of $4.29 \pm .63$ $\mathrm{s}$ for a carrying weight of $15 \%$. Post hoc testing confirmed the significant differences between the groups (Table 2).

Thus, gait analysis revealed that increased carrying weight was associated with decreases in stride length and step length and increases in step width and gait time, meaning that a greater carrying weight caused slower gait speeds in elderly women. Carrying a heavy weight (15\% of the mean body weight) in the WAV appeared to reduce gait efficiency.

\section{Effects of storage weight on gait analysis}

To confirm which factors in the gait pattern were most affected by carrying weight when using a WAV, we conducted simple regression analysis. In the case of stride length, the result showed that the unstandardized coefficient (b), which represents the influence of the model, was -.01, the coefficient of determination $\left(\mathrm{R}^{2}\right)$, which presents the explanatory power of the model, was .15 and the F-value, which presents the goodness of fit, was 14.93. Based on these results, the results were statistically significant $(\mathrm{p}<.001)$. For step length, the unstandardized coefficient (b) was -.03 , the coefficient of determination $\left(\mathrm{R}^{2}\right)$ was .08 and the F-value was 7.09 , indicating that the results were statistically significant $(\mathrm{p}<.01)$.

In the case of step width, the results showed that the unstandardized coefficient (b) was .20, the coefficient of determination $\left(\mathrm{R}^{2}\right)$ was .50 and the F-value was 87.68 , indicating that the results were statistically significant $(p<.001)$. In the case of gait time, the unstandardized coefficient (b) was .61, the coefficient of determination $\left(R^{2}\right)$ was .32 and the F-value was 41.92 , indicating that the results were statistically significant $(p<.001)$ (Table 3). Thus, carrying weight affected the gait of elderly women using a WAV and had the greatest effect on gait time.

\section{Discussion}

Because WAV are operated with two hands, they have luggage compartments for carrying possessions. This compartment should provide convenience without interfering with use of the WAV, as well as have a structure 
Table 2. Gait Analysis According to Storage Weight

\begin{tabular}{|c|c|c|c|c|}
\hline Classification & Average weight (\%) & Distance (cm) & $\mathrm{F}$ & Post Duncan \\
\hline \multirow{4}{*}{ Stride length } & $0 \%^{\mathrm{a}}$ & $76.16 \pm 19.70$ & \multirow{4}{*}{$6.73 * * *$} & \multirow{4}{*}{$\mathrm{a}, \mathrm{b}, \mathrm{c}>\mathrm{d}$} \\
\hline & $5 \%{ }^{b}$ & $77.19 \pm 17.47$ & & \\
\hline & $10 \%^{\mathrm{c}}$ & $69.70 \pm 21.57$ & & \\
\hline & $15 \%{ }^{\mathrm{d}}$ & $55.79 \pm 22.11$ & & \\
\hline \multirow{4}{*}{ Step length } & $0 \%{ }^{\mathrm{a}}$ & $36.86 \pm 9.10$ & \multirow{4}{*}{$6.42 * * *$} & \multirow{4}{*}{$a, b, c>d$} \\
\hline & $5 \%^{b}$ & $37.90 \pm 10.59$ & & \\
\hline & $10 \%{ }^{\mathrm{c}}$ & $33.42 \pm 8.16$ & & \\
\hline & $15 \%{ }^{\mathrm{d}}$ & $26.91 \pm 7.67$ & & \\
\hline \multirow{4}{*}{ Step width } & $0 \%{ }^{\mathrm{a}}$ & $12.49 \pm 2.26$ & \multirow{4}{*}{$42.80 * * *$} & \multirow{4}{*}{$\mathrm{a}, \mathrm{b}, \mathrm{c}<\mathrm{d}$} \\
\hline & $5 \%^{\mathrm{b}}$ & $11.48 \pm 2.14$ & & \\
\hline & $10 \%^{\mathrm{c}}$ & $15.17 \pm 2.14$ & & \\
\hline & $15 \%^{\mathrm{d}}$ & $16.75 \pm 2.15$ & & \\
\hline \multirow{4}{*}{ Gait time $(4.8 \mathrm{M} / \mathrm{sec})$} & $0 \%{ }^{\mathrm{a}}$ & $3.36 \pm .94$ & \multirow{4}{*}{$15.22 * * *$} & \multirow{4}{*}{$a>b>c>d$} \\
\hline & $5 \%{ }^{b}$ & $2.23 \pm .45$ & & \\
\hline & $10 \%^{\mathrm{c}}$ & $3.31 \pm .69$ & & \\
\hline & $15 \%{ }^{\mathrm{d}}$ & $4.29 \pm .63$ & & \\
\hline
\end{tabular}

$* * \mathrm{p}<.01, * * * \mathrm{p}<.001$

Table 3. Effects of Storage Weight on Gait Analysis

\begin{tabular}{|c|c|c|c|c|c|c|c|c|c|c|c|c|}
\hline \multirow{2}{*}{$\begin{array}{l}\text { Independent } \\
\text { variable }\end{array}$} & \multicolumn{3}{|c|}{ Stride length } & \multicolumn{3}{|c|}{ Step length } & \multicolumn{3}{|c|}{ Step width } & \multicolumn{3}{|c|}{ Gait time } \\
\hline & $\mathrm{b}$ & $\beta$ & $\mathrm{t}$ & B & $\beta$ & $\mathrm{t}$ & $\mathrm{b}$ & $\beta$ & $\mathrm{t}$ & $\mathrm{b}$ & $\beta$ & $\mathrm{T}$ \\
\hline Storage weight & -.01 & .38 & $-3.86 * * *$ & -.02 & -.27 & $-2.66^{* *}$ & .20 & .71 & $19.36^{* * *}$ & .61 & .57 & $6.47^{* * *}$ \\
\hline Constant & 2.96 & & $11.36^{* * *}$ & 2. & & $9.35 * * *$ & & & $2.88^{*}$ & & & -.58 \\
\hline $\mathrm{R}^{2}$ & \multicolumn{3}{|c|}{.15} & \multicolumn{3}{|c|}{.08} & \multicolumn{3}{|c|}{.50} & \multicolumn{3}{|c|}{.32} \\
\hline $\mathrm{F}$ & \multicolumn{3}{|c|}{$14.93 * * *$} & \multicolumn{3}{|c|}{$7.09 * *$} & \multicolumn{3}{|c|}{$87.68 * * *$} & \multicolumn{3}{|c|}{$41.92 * * *$} \\
\hline
\end{tabular}

$* * * \mathrm{p}<.001$

that prevents the WAV from overturning when carrying luggage. However, improper use of such compartments leads to increases in inappropriate control and therefore increased risk of collision increases (Park, 2017). Thus, this study conducted gait analysis to analyze changes in gait according to increases in the carrying weight using a WAV for elderly women.

Our study showed that increased carrying weight is associated with decreased stride length and step length and increased step width and gait time. In particular, negative effects on gait were observed in the group with a carrying weight equivalent to $15 \%$ of the mean body weight of elderly women in our study. These results are supported by findings from Kim and Lee (2013) and Hoyt et al. (2000), who reported that the effects of increased weight were mechanisms acting to minimize body tremor and load effects and to ensure stability. These mechanisms were reported to cause negative changes in gait, which increased 
the contact time between the foot and the ground, leading to slower gait time.

Studies by Kim and Lee (2013), Chow et al. (2005), and Hong and $\mathrm{Li}$ (2005) have also shown that external loads should be controlled so that they do not cause abnormal posture. When external loads are increased to $>15 \%$ of the body weight, adaptive mechanisms involving abnormal posture most likely occur, particularly in elderly women. Increased stress due to continual loads can lead to musculoskeletal damage; therefore, elderly women require more care than the elderly men. Thus, changes in the gait of elderly women with increased load when walking with a WAV are supported by the results of previous studies, and for the safety of elderly women using a WAV, the weight loaded into the luggage compartment of a WAV should not exceed $15 \%$ of their mean body weight.

Meanwhile, Jung (2009) reported that gait time, which is the result of changes in stride length, step length, and step width, is the important information to prevent the risk of falls by the elderly and can also provide information on how stability and function are affected by changes in time. In our study, gait time also increased with increased carrying weight and changed according to the changes in stride length, step length, and step width. These results are supported by Kim and Lee (2013), Jung (2009) and Lee (2007), who reported that gait time was strongly correlated with stride length, step length, and step width. Therefore, when using a WAV to minimize changes in gait time in safe outdoor movement, the appropriate carrying weight should be maintained.

In contrast, with a carrying weight of $5 \%$ of the mean body weight, the stride and step lengths increased, and the step width and gait time decreased. Jung et al. (2011) reported that the center of mass of the WAV is an important variable in its safe operation, and that appropriate positioning of the center of mass provides static and dynamic stability and is the most important factor during movement. This center of mass is affected by external loads; therefore, utilizing the appropriate load can effectively improve the maintenance of dynamic balance and stabilize gait because of its beneficial effects on static and dynamic stability of the trunk.

Jung et al. (2011) reported that using an appropriate weight reduces WAV tremors during straight-line travel, helping to increase stability. Although we did not observe a statistically significant difference in our study, we found that gait variables were most efficient when the WAV was loaded with a weight of $5 \%$ compared to when no weight was added $(0 \%)$. This shows that an appropriate weight in the WAV has a positive effect on gait by reducing the limitations accompanying physical activity (Shin et al., 2015). Specifically, using an appropriate weight positively lowers the center of mass of the WAV, thereby enhancing trunk stability, reducing tremor of the WAV during movement, and facilitating efficient, stable gait. Therefore, a suitable load should be used to improve the stability of the WAV during outdoor travel, to enhance gait efficiency, and to reduce the physical burden.

Generally, as aging progresses, the elderly have an increased risk of fracture due to a decrease in bone density. They are also easily influenced by extrinsic variables such as excessive force and heavy weight, which adversely affect their gait by decreasing their balance and stability. Moreover, the negative influence of these variables is greater in elderly women than elderly men (Chang, 2011). Thus, based on the results of this study, it is important to consider the application and management of an appropriate storage weight to improve the design of WAVs.

It should be noted that our study had several limitations. First, among various gait properties during walking with a WAV, we only analyzed variables relating to time and distance. Gait is a complex, dynamic, rhythmical movement, and its properties can be analyzed using several different variables. In future studies, the effects of weight on gait should be further investigated by analyzing the gait cycle, including the stance phase, swing phase, and 
double support phase, as well as changes in the angles of the ankles and feet. The luggage compartment of the WAV is located in the lower part, next to the wheels; therefore, changes in the movements of the upper and lower limbs should also be monitored during walking. Thus, there is a need for more in-depth analysis of gait changes with increased weight, including analysis of movements of the waist, hips, and knees.

\section{Conclusion}

In this study, we analyzed the effects of various carrying weights on gait in elderly women when using a WAV. We believe that this study has academic importance in that it will help determine the appropriate weight to increase gait stability loading for WAVs. We found that different carrying weights led to changes in gait and differences between weight groups. In particular, we saw the most positive effects on gait efficiency at 5\% carrying weight, and the greatest decrease in gait efficiency at $15 \%$ carrying weight. Therefore, when elderly women use a WAV for outdoor travel, loading a small amount of weight leads to greater stability than loading no weight; however, a weight of $<15 \%$ of the body weight should be used.

\section{References}

Baek SJ. A study on design of ambulatory aid car considering user's specificity. Master's Degree. Kookmin University. 2012.

Chang HS. Analysis of utilization and system development for prosthetic appliances and aid of the frail elderly people. Seoul in Korea: Korea Health Industry Development Institute. 2001;2011(1):1-221

Cho KH, Jang KB, Song MJ. Design improvement for the stability of walking aids (Rollators). J of HIC Soc of Kor. 2011;2011(1):915-8.

Chow DHK, Kwok MLY, Au-Yang ACK, et al. The effect of backpack load on the gait of normal adolescent girls. Ergo. 2005;48(6):642-56.

Hong Y, Li JX. Influence of load and carrying methods on gait phase and ground reaction in children's stair walking. Gait \& Post. 2005;22(1):63-8.

Hoyt DF, Wickler SJ, Cogger EA. Time of contact and step length: The effect of limb length, running speed, load carrying and incline. J of Exp Bio. 2000; 203(2):221-8.

Jung HS. A study of muscle activities and gait analysis between young adults and elderly people according to gait speed in level walking. Doctor's Degree. Dague University. 2009.

Jung JS, Ahn YH, Hwang IH, et al. Evaluation on Wheelchair Driving Performance based on its Center of Gravity. The Kor Soc of Manu Proc Eng. 2011;2011(11):93.

Jung KT, Shin DJ, Chun KJ, et al. Anthropometric analysis and usability evaluation of four-wheeled walker. $\mathrm{J}$ of the Ergo Soc of Kor. 2009;28(2):17-26.

Kim CK, Lee BH. Gait analysis according to the changes of the carrying type and weight of bag. $\mathrm{J}$ of the Kor Aca Indu coo Soc. 2013;14(1):199-205.

Lee KS. Analysis of temporal and spatial gait parameters of male and female in 20s and 60s. J of Kor Phys Edu Asso for Girls and Wom. 2007;21(4):55-66.

Lee SH, Kim BM, Lee WY, et al. A study on the risk sensing method for walking assistant vehicle over irregular terrain. J of Rehab Welf Eng \& Asso Tech. 2013; 2013(4):113-6.

Park ER. A Study on the active aging environment for the aged who need four-wheeled walker with seat: Focused on the residents in Dobon 1 dong. Master's Degree. Seoul National University. 2017.

Park JH. Effects of the development and application of an intervention program for the prevention of fractures for elderly women with osteopenia. Doctor's Degree. Daegu Catholic University. 2018.

Park JY, Cho A. A safety evaluation of walking aids for the 
aged. J of Ergo Soc of Kor. 2008;2008(1):1-5.

Park MS, Cho SH, Shin SH, et al. Comparison of gait stability of walking used an outdoor rollator with walking used a bassinet as ambulatory aid. J of Ergo Soc of Kor. 2009;2009(11):42-53.

Roh HL, Shin EJ. Gait analysis of walker depending external loads. J of the Kor Aca Indu coo Soc. 2015;2015(1): 631-4.

Shin EJ, Jeon BJ, Song BK, et al. Analysis of walker-aided walking by the healthy elderly with a walker pocket different weights attached at different location. $\mathrm{J}$ of Phys Ther Sci. 2015;27(11):3369-71. 